\title{
O IMPACTO DA PANDEMIA DA COVID-I9 NA FORMAÇÃO MÉDICA: UMA REVISÃO INTEGRATIVA
}

\author{
Bianca Mendes Coelho ${ }^{1}$ \\ Erick Brito Meira ${ }^{2}$ \\ Liara Souza de Araújo ${ }^{3}$ \\ Paula Teixeira Fernandes de Araújo ${ }^{4}$ \\ Sthéfani Serra Tenisi ${ }^{5}$ \\ Ana Carolina Soares Rios Santos 6 \\ Daniela Neves Dourado ${ }^{7}$ \\ Maria Eduarda Visniewski da Cunha ${ }^{8}$ \\ Mariana Rios Vilas Boas9 \\ Matheus Benamor Duarte ${ }^{10}$ \\ Roberto de Barros Silva ${ }^{11}$
}

RESUMO: A pandemia gerada com a disseminação do vírus Sars-Cov-2 e o consequente isolamento social reconfiguraram o modo de ensino tradicionalmente presencial para o modelo remoto. O objetivo deste estudo é avaliar as implicações desse novo modelo na formação dos futuros médicos a partir de uma revisão integrativa. Assim, a pesquisa tem caráter qualitativo e foi realizada por meio da busca de artigos indexados nas bases de dados Publisher Medline (PubMed) e Scientific Eletronic Library Online (SciELO), utilizando os seguintes descritores: "COVID-ı"”; "Pandemia COVID-ı"”; "Infecção pelo SARS-CoV-2"; "Medicina"; "Medicine"; "Educação à distância"; "Education. Distance"; "Estudantes de Medicina"; "Students, Medical”. Foram encontrados 133 artigos (n=133) que, após a aplicação dos critérios de inclusão e exclusão, foram reduzidos para 8o artigos $(n=80)$ os quais, após análise por leitura integral, foram reduzidos para 54 artigos $(n=54)$. Ainda não ficou claro o impacto que esse novo modelo de ensino causará a longo prazo na formação médica, no entanto, é imprescindível que o ensino remoto e o currículo das escolas médicas sejam aperfeiçoados devido a continuidade da pandemia da COVID-19 ou outros eventos que afetem a interação social e, consequentemente, o ensino médico presencial.

\footnotetext{
${ }^{1}$ Acadêmica do curso de Medicina, sexto período, da Universidade Salvador (UNIFACS)

${ }^{2}$ Acadêmico do curso de Medicina, sexto período, da Universidade Salvador (UNIFACS)

${ }^{3}$ Acadêmica do curso de Medicina, sexto período, da Universidade Salvador (UNIFACS

${ }^{4}$ Acadêmica do curso de Medicina, sexto período, da Universidade Salvador (UNIFACS)

${ }^{5}$ Acadêmica do curso de Medicina, sexto período, da Universidade Salvador (UNIFACS)

${ }^{6}$ Acadêmica do curso de Medicina, sexto período, da Universidade Salvador (UNIFACS)

${ }^{7}$ Acadêmica do curso de Medicina, sexto período, da Universidade Salvador (UNIFACS)

${ }^{8}$ Acadêmica do curso de Medicina, sexto período, da Universidade Salvador (UNIFACS)

${ }^{9}$ Acadêmica do curso de Medicina, sexto período, da Universidade Salvador (UNIFACS)

${ }^{10}$ Acadêmica do curso de Medicina, sexto período, da Universidade Salvador (UNIFACS)

${ }^{\text {II }}$ Graduado em Biomedicina; doutor em Farmacologia pela King'sCollege London; pós doutor em Farmacologia pela ICB-USP; docente do curso de Medicina da Universidade de Salvador (Unifacs - BA)
} 

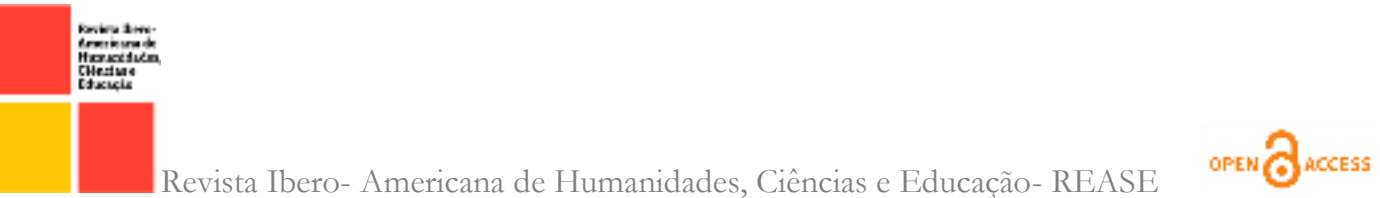

Palavras-chaves: Ensino remoto. Graduação em medicina. educação à distância. Telemedicina. Tandemia do COVID-I9.

ABSTRACT: The pandemic of the Sars-Cov-2 virus and the isolation caused by it reconfigured the traditional face-to-face teaching method for the remote model. This study aims to analyze the implications of this change in the training of future physicians using an integrative review. The research has a qualitative character and was made through the search for articles indexed in the Publisher Medline (PubMed) and Scientific Electronic Library Online (SciELO) databases, using the following descriptors: "COVIDI9"; "COVID-I9 pandemic"; "SARS-CoV-2 infection"; "Medicine"; "Medicine"; "Distance Education"; "Education. Distance"; "Medical students"; "Students, Medical”. I33 articles were found and analyzed based on the inclusion and exclusion criteria. After this process, the number of articles was reduced to 80. Those were fully read and reduced again, now to 54 articles. It is still unclear what impact the new model of education will have on medical training in the long term. However, it is essential that remote teaching and the curriculum of medical schools continue to be improved due to the persistence of the COVID-I9 pandemic or the possibility of other events that affect social interaction, causing online medical education.

Keywords: Remote Teaching. medical degree. distante education. Telemedicine. COVID19 pandemic.

\section{INTRODUÇÃO}

Identificado em dezembro de 2019, o vírus Sars-Cov-2, causador de um espectro de sintomas, desde uma síndrome gripal a uma síndrome respiratória aguda grave, foi responsável por uma série de casos na China que logo se expandiu para uma crise sanitária global, sendo decretado, em março de 2020, estado de pandemia pela Organização Mundial da Saúde (OMS) ${ }^{12}$. Nesse cenário, a sociedade foi submetida a inúmeros desafios, um destes tem sido a continuidade do aprendizado dos alunos em todos os graus de ensino diante da impossibilidade da interação presencial, sendo a formação médica uma das mais afetadas.

A educação médica sempre teve suas peculiaridades e desafios ${ }^{\mathrm{I}}$ : os conhecimentos

\footnotetext{
${ }^{12}$ https://www.unasus.gov.br/noticia/organizacao-mundial-de-saude-declara-pandemia-de-coronavirus.

${ }^{13}$ CAMPOS, Maria Angélica de Figueiredo; FORSTER, Aldaísa Cassanho. Percepção e avaliação dos alunos do curso de medicina de uma escola médica pública sobre a importância do estágio em saúde da família na sua formação. Rev. bras. educ. med., Rio de Janeiro, v. 32, n. I, p. 83-89, Mar. 2008. SANTOS, Bruna Mascarenhas et al. Educação Médica durante a Pandemia da Covid-rg: uma Revisão de Escopo. Rev. bras. educ. med., Brasília, v. 44, supl. I, e139, 2020.
} 
técnicos e científicos, a humanização da relação médico-paciente e os aspectos psicológicos e emocionais intrínsecos à formação médica. Diante do estado epidemiológico, a impossibilidade de interações interpessoais tem sido o maior obstáculo na manutenção do aprendizado em um curso majoritariamente prático, no qual o contato é imprescindível.

Para o alcance desse conjunto de habilidades e competências, os métodos pedagógicos utilizados historicamente na educação médica são baseados em relações interpessoais, as quais foram afetadas pela interrupção do processo de ensino-aprendizagem presencial causada pela pandemia da Covid-19. (SANTOS, 2020)

No contexto do isolamento social imposto pela pandemia, a educação também passou por adaptações. Salas virtuais, avaliações online e práticas remotas se tornaram realidade no processo de aprendizado dos futuros médicos. Apesar de suprir algumas demandas, essa metodologia de ensino ainda tem grandes limitações, visto que a Medicina é um fazer no qual é imperioso o uso das habilidades sensórias - ver, tocar, sentir, ouvir. Nesse sentido,

"o afastamento de alunos de estágios clínicos pode ter implicações significativas para o planejamento futuro da força de trabalho" (MORETTI-PIRES et al, 202I).

Ademais, sendo a formação acadêmica composta pelos componentes teóricos e práticos, a suspensão das atividades práticas presenciais produz nos estudantes um sentimento de ansiedade sobre a incerteza da consolidação dos conhecimentos e sobre quando essas limitações serão sanadas .

Assim, este estudo tem como objetivo analisar o impacto do ensino remoto na formação médica, como também investigar quais as estratégias pedagógicas estão sendo utilizadas e se essas vem suprindo o aprendizado prático-presencial, como a relação médico-paciente e o treinamento das habilidades de comunicação.

\section{METODOLOGIA}

O estudo trata-se de uma revisão integrativa sobre a temática: $O$ impacto da pandemia da covid-ı9 na formação médica. Essa metodologia consiste em um estudo bibliográfico, de caráter qualitativo em que o mais importante é a interpretação por parte 
do pesquisador com suas opiniões sobre o fenômeno em estudo (PEREIRA et al, 2018 apud SOARES NETO et al, 202I).

No presente estudo, a revisão integrativa foi realizada em seis etapas: Definição da pergunta da revisão; busca e seleção dos estudos primários; extração de dados dos estudos primários; avaliação crítica dos estudos primários; síntese dos resultados da revisão; apresentação da revisão, como mostra a figura I abaixo:

Figura I: Etapas da revisão integrativa, Ribeirão Preto, São Paulo, 2017 (apud MENDES, SILVEIRA, GALVÃO, 2019)

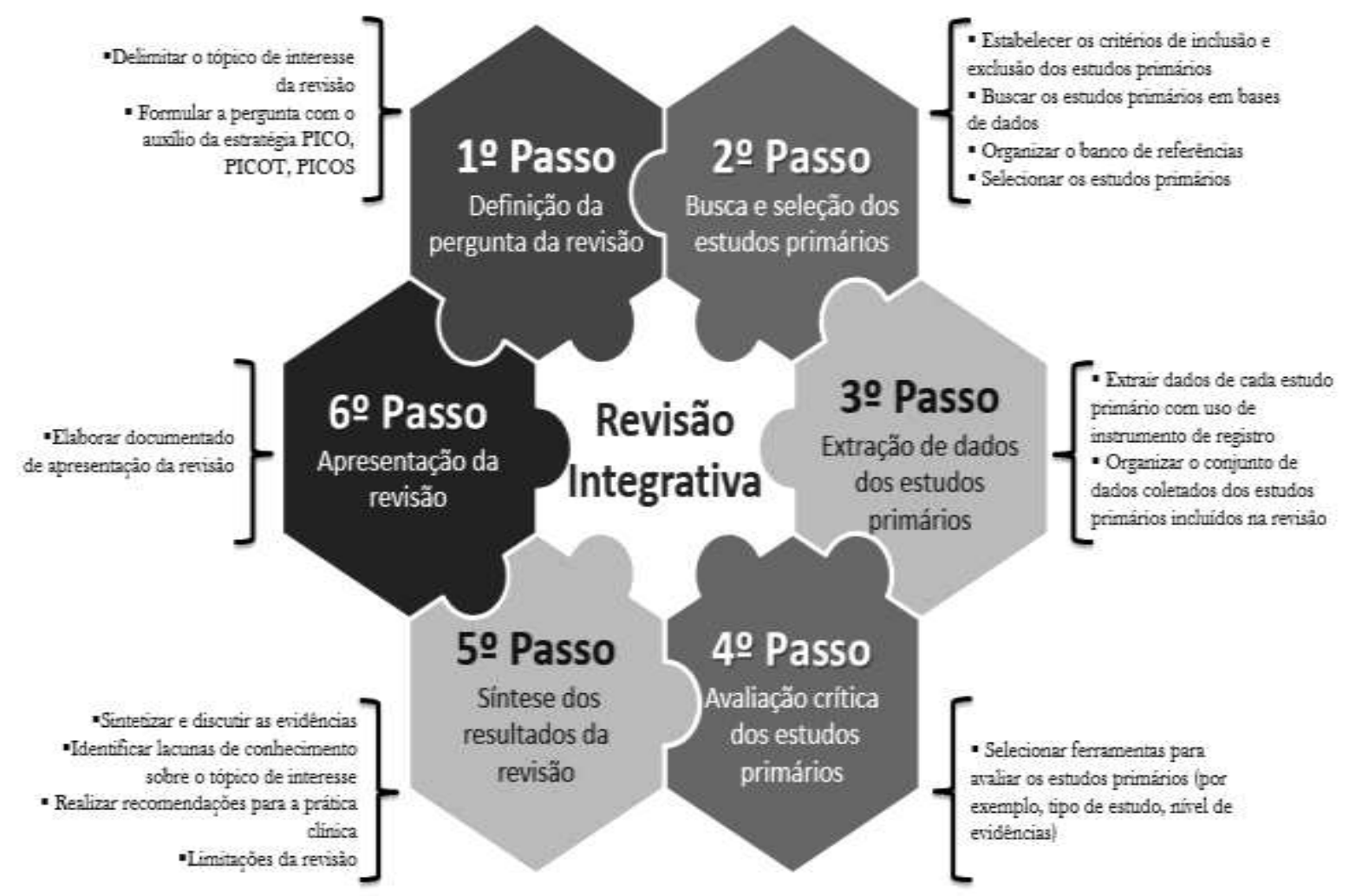

Diante da delimitação da problemática e definição dos objetivos, foram estabelecidos os descritores para o refinamento da busca dos artigos primários. Tais descritores, determinados através da plataforma Descritores em Ciências da Saúde (DECS) foram: "COVID-ı9"; "Pandemia COVID-ı9”; "Infecção pelo SARS-CoV-2"; 
"Medicina"; "Medicine"; "Educação à distância"; "Education. Distance"; "Estudantes de Medicina"; "Students, Medical". As bases de dados utilizadas foram Publisher Medline (PubMed) e Scientific Eletronic Library Online (SciELO).

Essa busca resultou na seleção de I3I artigos na base de dados PubMed e 2 artigos no SciELO. Então, com a definição dos critérios de inclusão (a partir de 2020; idiomas português e inglês; durante a graduação de Medicina; ensino remoto no contexto da pandemia) e critérios de exclusão (anteriores a 2020; em outro idioma que não português e inglês; em outros momentos que não a graduação médica; ensino remoto de outras áreas e fora do contexto da pandemia).

Depois de aplicados os critérios de inclusão e exclusão, restaram 80 artigos elegíveis. Esses passaram por uma leitura integral e na análise dos respectivos critérios previamente definidos para esta revisão, permitindo a exclusão de 26 artigos. Assim, os estudos incluídos totalizam 54 artigos.

Para a extração de dados, foram elaborados 4 questionamentos como norteadores (Tabela I) de forma a responder os objetivos do presente estudo. Segue fluxograma do processo de seleção dos estudos (Figura 2).

Tabela I

\begin{tabular}{|l|l|}
\hline \multicolumn{2}{|c|}{ QUESTIONAMENTOS NORTEADORES } \\
\hline Questionamento I & $\begin{array}{l}\text { Como foi feita a adaptação ao ensino remoto, englobando as } \\
\text { estratégias pedagógicas? }\end{array}$ \\
\hline Questionamento 2 & $\begin{array}{l}\text { Quais as dificuldades encontradas para adaptação frente ao ensino } \\
\text { remoto? }\end{array}$ \\
\hline Questionamento 3 & $\begin{array}{l}\text { As estratégias pedagógicas e práticas de telemedicina vem } \\
\text { suprindo o aprendizado prático-presencial? }\end{array}$ \\
\hline Questionamento 4 & O que a pandemia deixa para o futuro da formação médica? \\
\hline
\end{tabular}


Figura 2: Representação do processo de seleção dos estudos

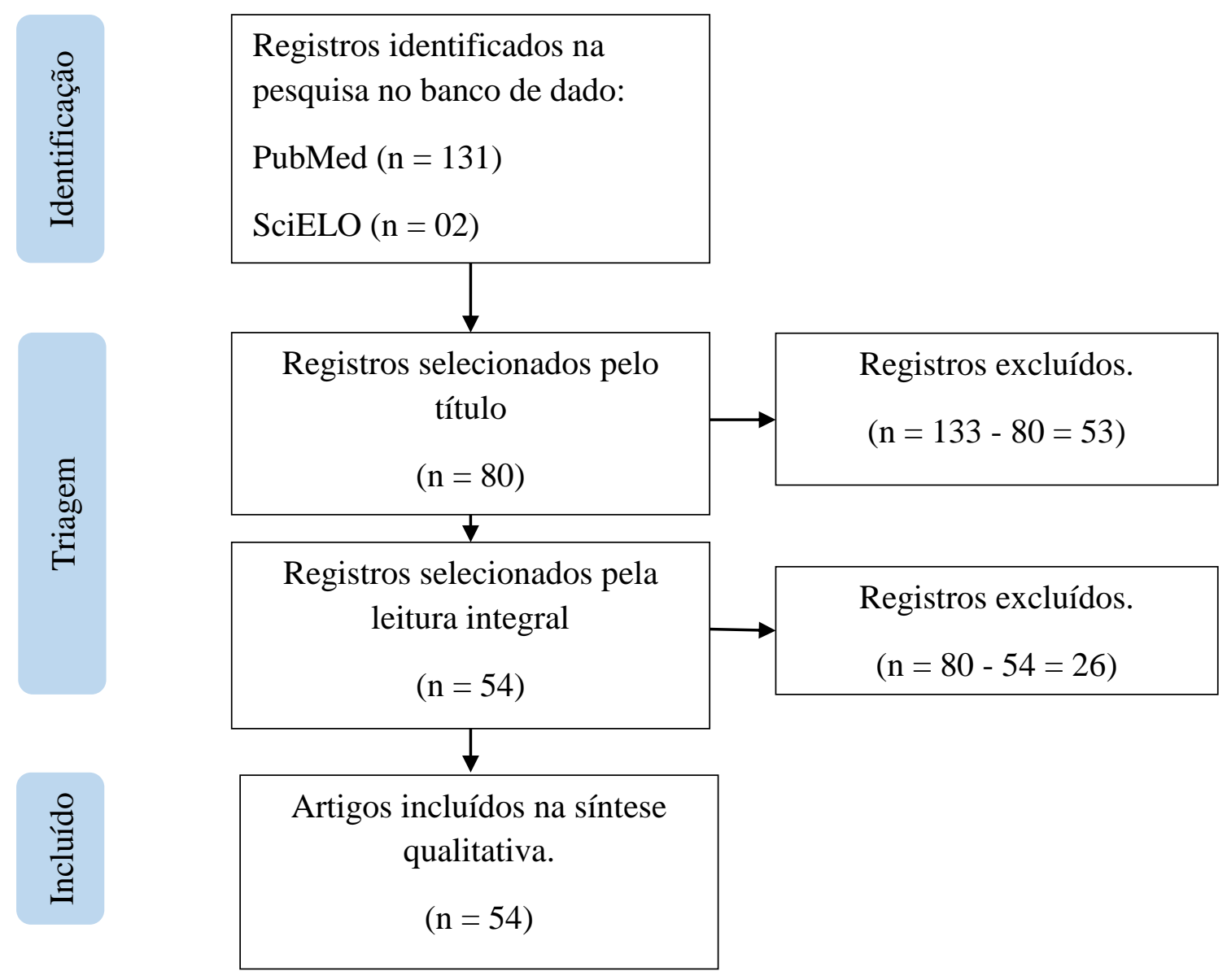

\section{ESTRATÉGIAS PEDAGÓGICAS: ADAPTAÇÃO AO ENSINO REMOTO}

A educação médica foi afetada diretamente em vigência da pandemia da COVIDI9, o que provocou a interrupção de diversas atividades acadêmicas, como a suspensão das aulas presenciais e estágios clínicos. Assim, as faculdades de Medicina foram impelidas a buscar diferentes métodos de ensino que permitissem uma continuação do processo de aprendizagem.

Foi possível constatar, por meio de 54 estudos incluídos na revisão integrativa, que houve uma mudança tanto no aspecto pedagógico quanto no aspecto avaliativo, no que tange o ensino médico. Desses artigos, 47 respondem ao questionamento "Como foi feita a 
adaptação ao estudo remoto? (Estratégias pedagógicas)" (Gráfico I), sendo a utilização de plataformas digitais - Zoom, Blackboard, Hangouts, Skype, Microsoft Teams - a principal estratégia empregada, com finalidade de prosseguir com o ensino. Assim, os professores realizam videoconferências, de modo síncrono ou assíncrono, permitindo que os alunos tenham acesso às aulas por meio de computadores, tablets e celulares.

(Gráfico I)

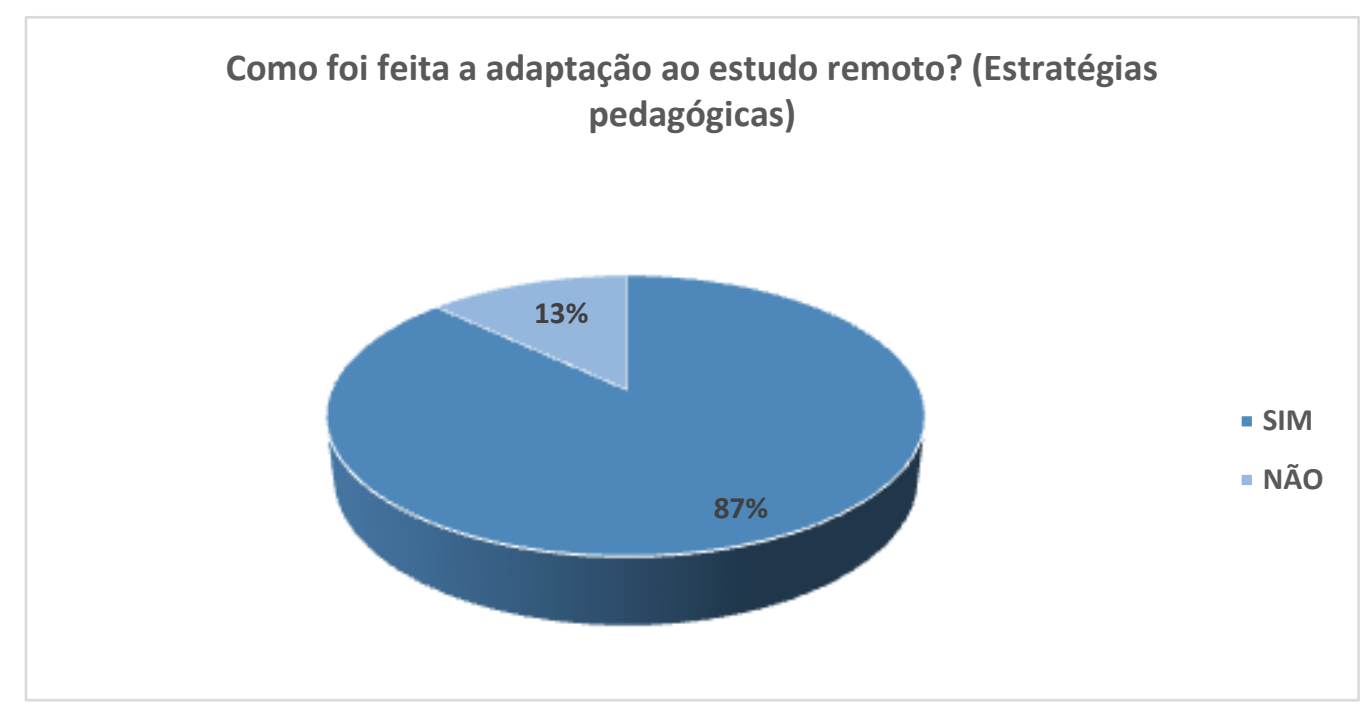

Conforme Oladipo et al (2020), outra abordagem metodológica recorrida têm sido o uso de softwares de telemedicina, nos quais os estudantes conseguem participar de consultas médicas, mesmo que à distância. Assim, os futuros médicos conseguem desenvolver o raciocínio clínico e escolha da terapêutica adequada, remotamente. Evidencia-se, portanto, que essa estratégia pedagógica visa uma tentativa de suprir a deficiência dos alunos no tocante ao exercício de atividades práticas, tendo em vista de que é um dos aspectos mais prejudicados do ensino devido à dificuldade de transpor esse treinamento para o ambiente online.

Outrossim, estudos como "Changes in undergraduate medical education due to COVID-I9: a systematic review", revelam o uso de metodologias de estudo ativo, como o Team Based Learning (TBL) e Problem Based Learning (PBL), que proporcionam a criação de salas de aula invertidas, nas quais os estudantes ocupam um espaço de autonomia na construção do conhecimento. Logo, essa proposta possibilita uma interação mais participativa dos alunos dentro desse cenário do ensino remoto. 
Logo, é possível observar que muitas faculdades têm adotado estratégias pedagógicas através da tecnologia que permitam transferir o conteúdo teórico para o ambiente digital, enquanto o currículo prático - componente imprescindível na formação médica - tem sido adiado para o período em que as atividades presenciais estejam estabelecidas novamente.

\section{AS DIFICULDADES NA ADAPTAÇÃO AO ENSINO REMOTO}

Foi observado, a partir da análise dos 54 artigos, que 39 desses respondem ao questionamento "Quais as dificuldades encontradas para a adaptação ao ensino remoto?" (Gráfico 2). Tais estudos revelam que houve muitas dificuldades comuns à maioria dos estudantes de medicina durante o período em que as aulas presenciais foram substituídas por aulas remotas. Os problemas variaram desde questões técnicas relacionadas ao uso dos softwares, plataformas e conexão com a internet, a problemas envolvendo a saúde mental dos discentes e a dinâmica do ambiente em que eles estavam inseridos durante o período de isolamento social (Tabela r).

(Gráfico 2)

Quais as dificuldades encontradas para a adaptação frente ao ensino online?

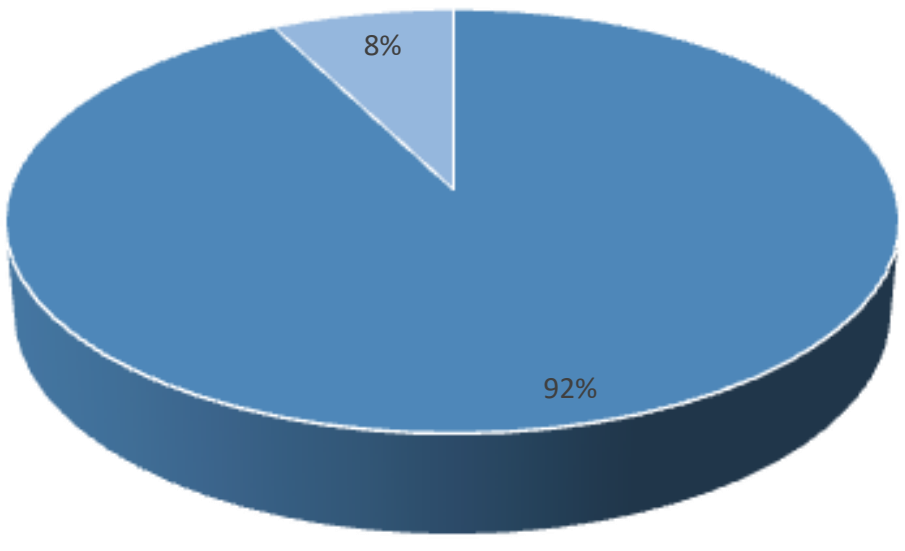

- SIM

- NÃO 
(Tabela I)

\begin{tabular}{|l|l|}
\hline DIFICULDADE ENCONTRADA & NÚMERO DE ARTIGOS \\
\hline Falta de interação (colegas, professores e pacientes) & 15 \\
\hline $\begin{array}{l}\text { Dificuldades técnicas (design, desenvolvimento de } \\
\text { programas, conexão e velocidade da internet) }\end{array}$ & 13 \\
\hline Falta de atividades práticas & 9 \\
\hline Falta de interesse dos alunos & 9 \\
\hline Problemas de saúde e doenças psicológicas & 8 \\
\hline Dificuldades financeiras & 6 \\
\hline Dificuldade de comunicação & 5 \\
\hline Adaptação do corpo docente & 5 \\
\hline Ambiente (distrações, falta de espaço) & 4 \\
\hline Carga horária & 4 \\
\hline $\begin{array}{l}\text { Ausência de recursos institucionais adequados (equipe de TI, } \\
\text { plataformas eficazes, etc) }\end{array}$ & 3 \\
\hline $\begin{array}{l}\text { Garantia de aprendizado e dificuldade de aplicação de } \\
\text { exames }\end{array}$ & 3 \\
\hline Disciplina & 2 \\
\hline Dificuldade de concentração & 1 \\
\hline Preocupação com o desenvolvimento de habilidades & 1 \\
\hline Descolamento interno de estudantes devido a guerras & 1 \\
\hline
\end{tabular}

A garantia de acesso à internet de qualidade e a oferta de plataformas de ensino eficazes não ocorreu de forma igualitária entre os estudantes. No Brasil, por exemplo, como explicitado por um dos artigos selecionados, "observou-se que as universidades privadas foram capazes de continuar com a implantação de plataformas eletrônicas que não estavam disponíveis para as instituições públicas." (OLADIPO et al, 2020). A partir desse primeiro exemplo, observa-se a desigualdade existente entre os estudantes das universidades privadas e os estudantes das universidades públicas. Enquanto os primeiros, em sua maioria, tiveram acesso a plataformas digitais eficientes logo no início da transição para o ensino remoto, os outros não puderam lograr desse mesmo direito.

Outrossim, a falta de uma conexão com a internet com qualidade suficiente para a transmissão de aulas e palestras foi um problema frequentemente encontrado nos artigos analisados. Esse impasse estava relacionado tanto a questões financeiras, quanto à localização da casa dos alunos. Na Nigéria, por exemplo, o maior obstáculo era inserir estudantes em vulnerabilidade social no ensino remoto, devido ao alto custo dessa transição e a um serviço de internet não confiável (OLADIPO et al, 2020). Por outro lado, no Paquistão, uma das reclamações comuns estava relacionada ao local de residência dos 
discentes, como explicitou Farooq e colaboradores (2020): "estudantes residentes em áreas rurais muitas vezes reclamam de problemas com transmissão ao vivo de palestras e com videoconferências para palestras.”

Ademais, alguns artigos trouxeram reclamações acerca da saúde mental dos estudantes. De fato, devido ao isolamento social e a imposição do ensino remoto para a continuidade das aulas, os estudantes ficaram sem o convívio proporcionado pelo ensino presencial, o que, por consequência, provocou o sentimento de solidão em muitos deles. Foram encontrados relatos sobre sintomas de ansiedade, depressão, angústia, solidão e falta de motivação em alguns artigos escolhidos para esta revisão. Além disso, "foram relatadas preocupações com o desenvolvimento inadequado de habilidades devido à suspensão de colocações em hospitais, ambiguidade em relação às perspectivas futuras e subsequentes implicações financeiras" (SHARMA, D; BHASKAR, S., 2020).

Também foram encontrados, dentro dos artigos selecionados, problemas acerca da dinâmica do ambiente em que o estudante estava inserido. Em um estudo envolvendo 272I estudantes de medicina do Reino Unido, 26,76\% reclamaram das distrações no ambiente familiar e $\mathrm{II}, 03 \%$ relataram sobre a falta de espaço como barreiras para o ensino online eficaz (DOST et al, 2020). Fica evidente, portanto, que a falta de um ambiente adequado foi um problema que prejudicou uma parte dos alunos durante o período de ensino remoto.

Além dessas, diversas outras dificuldades foram relatadas, como a falta de interação com os pacientes, um problema relatado por $70 \%$ dos entrevistados em uma pesquisa com estudantes de medicina poloneses (BACZZEK et al, 202I); ausência de oportunidades de observação ou auxílio em procedimentos (ASHRAFZADEH et al, 202I) e "oportunidades perdidas de aprendizagem suplementar, como palestras, atividades em pequenos grupos e simulações" (TRIEMSTRA et al, 202I).

\section{AS ESTRATÉGIAS PEDAGÓgICAS E PRÁtICAS DE TELEMEDICINA: BENEFÍCIOS E LIMITAÇÕES}

A partir do levantamento realizado, 26 dentre os 54 artigos responderam à pergunta "As estratégias pedagógicas e práticas de telemedicina vêm suprindo o aprendizado práticopresencial?” (Gráfico 3). 
(Gráfico 3)

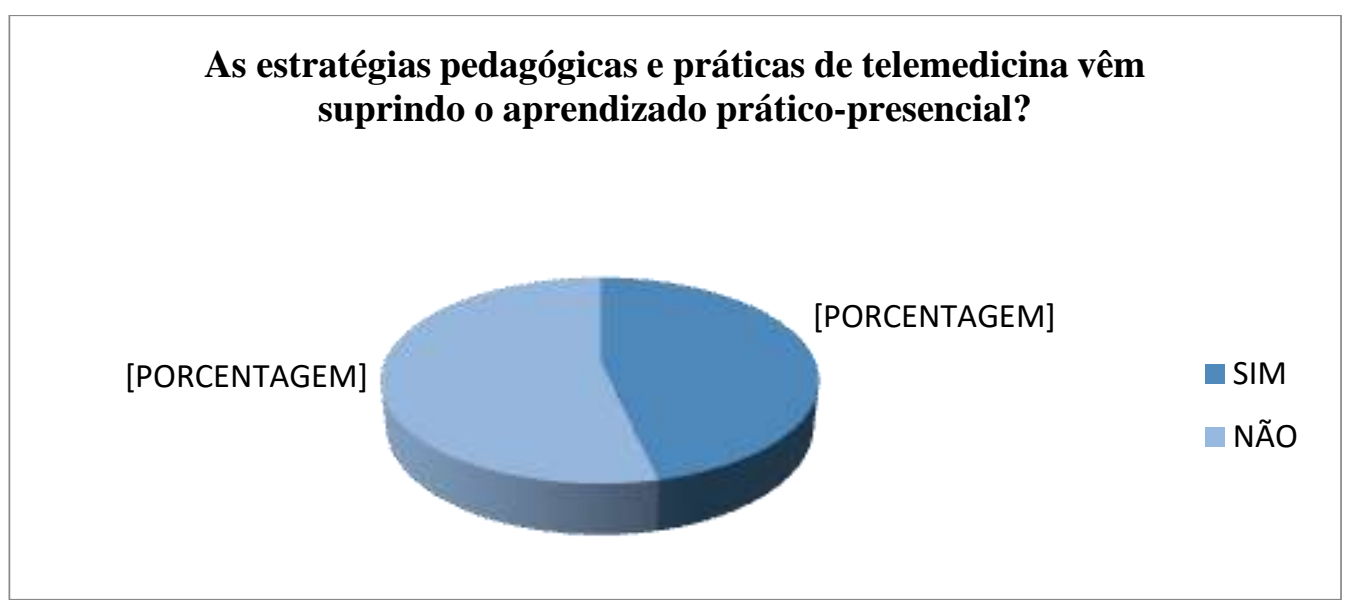

Diante desses resultados, é possível inferir que de maneira geral o ensino à distância foi bem aceito pelos estudantes de medicina. Em muitos artigos, o principal ponto levantado em relação à experiência virtual positiva diz respeito às aulas gravadas e uso da telemedicina. Sendo um consenso positivo compartilhado pelos alunos em relação a palestras pré-gravadas, a possibilidade de rever os conteúdos e reassistir os aspectos não compreendidos (CUSCHIERI; AGIUS, 2020). Além disso, foi observado que a 532 telemedicina "pode oferecer um meio único de envolver os alunos, proporcionando experiências autênticas de atendimento ao paciente e deve ser valorizada" (OSBORNE et al, 2021).

Ademais, o ensino à distância viabilizou o processo de autonomia do estudante, tornando-o um agente responsável também pelo seu próprio aprendizado, além de abrir espaço para a introdução das tecnologias digitais de informação e comunicação (MAGALHÃES et al, 2020). Essa autonomia estimulada também se mostra como uma característica imprescindível na formação de um bom profissional, além de potencializar a interação entre os pares.

Em contrapartida, houve uma insatisfação significativa também de boa parte dos estudantes, sobretudo em relação à falta do contato com o paciente e o déficit no desenvolvimento de habilidades clínicas, assim os mais afetados foram os alunos do ciclo clínico.

Segundo o estudo realizado no Reino Unido em maio de 2020 com 2.721 estudantes de medicina, 75,99\% ( $\mathrm{n}=\mathrm{I} 842$ ) tiveram a sensação que o ensino on-line não havia 
substituído com sucesso o ensino clínico recebido via contato direto com o paciente e $82,17 \%(n=1986)$ sentiram que não podem aprender habilidades clínicas práticas através do ensino online (DOST et al, 2020). Demonstrando que as habilidades clínicas são o principal déficit do ensino remoto, tornando-se uma barreira na adaptação do currículo ao modelo online.

\section{REPERCUSSÕES DA PANDEMIA PARA O FUTURO DA FORMAÇÃO MÉDICA}

Dos 54 artigos utilizados nesta revisão, apenas 3I deles respondem à pergunta: "O que a pandemia deixa para o futuro da formação médica?” (Gráfico 4). De modo geral, as restrições de ensino causadas pela Covid-ı9 foram percebidas como a abertura de oportunidades para novas experiências de aprendizagem para alunos e professores (BEHRENDS et al, 2020), sendo uma potente oportunidade para transformação digital da formação médica (HERRMANN-WERNER et al, 202I).

(Gráfico 4)

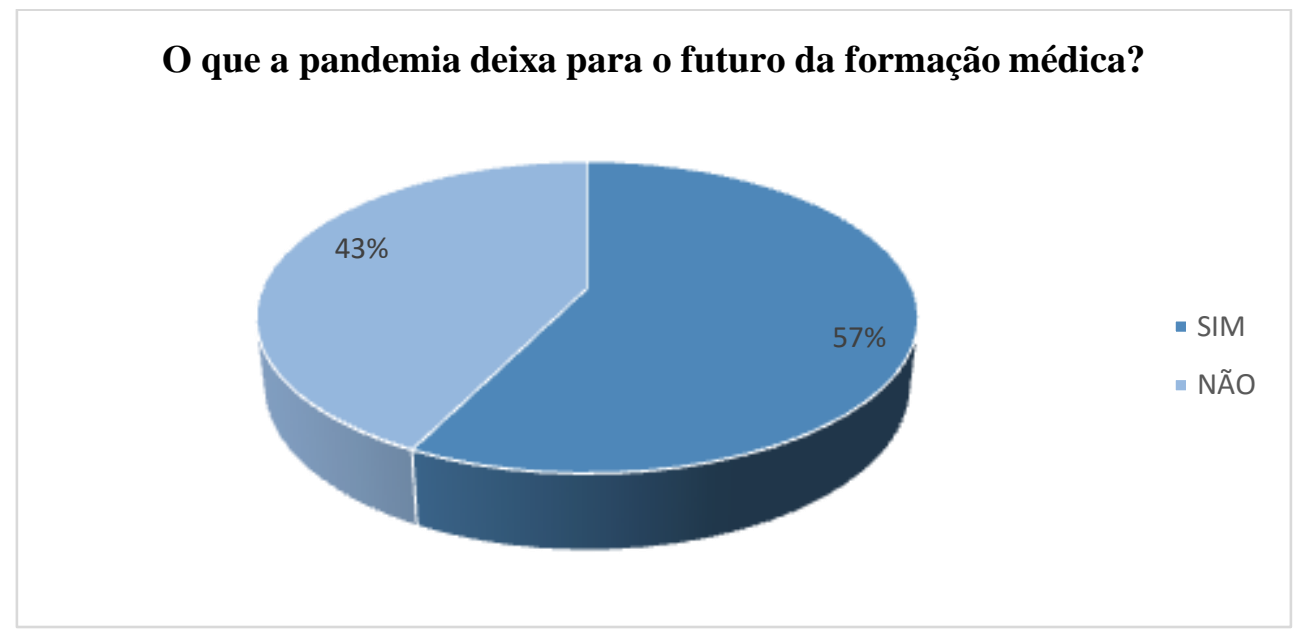

Para $\mathrm{Hu}$, Xiao e Li (202I) a pandemia trouxe novos olhos sobre as diversas tecnologias, e colocou em prova os diferentes métodos que podem ser usados para aprimorar o ensino médico. Nesta linha, a alta satisfação dos alunos e a experiência positiva ressaltadas em alguns artigos - incluindo maior flexibilidade, oportunidades para explorar diferentes recursos de aprendizagem e tempo para se concentrar no bem-estar com o ensino eletrônico a distância imposto pelo COVID-ı9 podem induzir mudanças 
futuras nos estudos médicos pré-clínicos, consolidando-se no currículo da formação médica (SANDHAUS et al, 2020; SHAHRVINI et al, 2021).

$\mathrm{Na}$ pesquisa realizada por Bączek e colaboradores (2021) os entrevistados acreditam que o ensino remoto é eficaz para aumentar o conhecimento e é altamente aceito, mas que este não deve focar apenas no aumento do conhecimento e na entrega de conteúdo, mas também nas habilidades clínicas e sociais e na capacidade dos alunos de trabalhar com os materiais e receber feedback. "A implementação bem-sucedida do aprendizado online no currículo requer uma estratégia bem pensada e uma abordagem mais ativa" (BĄCZEK et al, 2021).

O momento do curso de medicina - se ciclo básico (pré-clínico) ou ciclo clínico foi considerado de fundamental importância para se ponderar o que a pandemia deixa para formação médica. Assim, muitos dos artigos consultados concordam que:

\begin{abstract}
A aprendizagem combinada é o futuro da educação de profissionais de saúde. As atividades educacionais virtuais permitem a flexibilidade do aluno e do corpo docente (por exemplo, redução do tempo de deslocamento, disponibilidade global), enquanto as interações face a face garantem interações sociais $e$ avaliações das habilidades de atendimento direto ao paciente dos alunos" (TRIEMSTRA et al, 202I)
\end{abstract}

Desta forma, para Sukumar e colaboradores (202I) a implementação do currículo no ensino remoto e "as lições aprendidas com sua concepção e implementação, podem continuar a ter uma função de complementar na educação médica, mesmo após a retomada dos estágios presenciais”.

\title{
CONCLUSÃO
}

A pandemia da COVID-ı́ impôs um remodelamento abrupto na forma de ensino do currículo médico. Ao longo do estudo, através das perguntas definidas e dados elencados, foi possível observar que muitas faculdades têm adotado estratégias pedagógicas através da tecnologia que permitam transferir o conteúdo teórico para o ambiente digital, enquanto o currículo prático - componente imprescindível na formação médica - tem sido adiado para o período em que as atividades presenciais estejam estabelecidas novamente. Ficam claras, diante do exposto, as inúmeras dificuldades às quais os estudantes de medicina encontraram para adaptar-se ao ensino remoto durante a pandemia da COVID19, desde problemas técnicos de conexão com a internet, diferenças socioeconômicas ao 
acesso do mundo digital, a problemas que envolviam o cotidiano familiar dos discentes. Além disso, tornou-se evidente a falta de tempo hábil para a capacitação dos docentes em relação ao uso das ferramentas digitais e para a produção de materiais e conteúdos condizentes com a realidade online.

Deste modo, também foi observado que apesar dos desafios encontrados, houve avanços metodológicos durante a pandemia de COVID-i9, que desempenharam um papel particularmente importante na evolução pedagógica; mostrando que o método online pode ser uma ferramenta eficiente para o aprendizado. Contudo, fica claro que o modelo virtual não pode substituir as práticas clínicas, o contato direto com o paciente de maneira satisfatória e a perda das competências obtidas nessa interação, como por exemplo, o desenvolvimento das habilidades comunicativas e o entendimento da complexidade acerca do cuidado.

Dado o exposto, ainda é cedo para saber o impacto real a longo prazo da pandemia sobre as lacunas educacionais deixadas pela pandemia e o ensino remoto nos estudantes de medicina (DEDEILIA et al, 2020). Visto que a maioria dos estudos sobre formação médica, ensino remoto, COVID-ı9 descreveram até o momento estágios iniciais da mudança, e assim provar seus efeitos a longo prazo tem suas limitações (LEE et al 2021). Logo, se faz imperativo o aperfeiçoamento do ensino remoto e adaptação curricular da formação médica em virtude da continuidade da pandemia COVID-i9 e possíveis cenários que imponham novas restrições ao ensino médico presencial.

\section{REFERÊNCIAS}

AGYEI-NKANSAH, Adwoa; ADJEI, Patrick; TORPEY, Kwasi. COVID-I9 and medical education: an opportunity to build back better. Ghana Medical Journal, v. 54, n. 4s, p. I13II6, 2020. Acesso em: 12 de novembro de 202I < https://pubmed.ncbi.nlm.nih.gov/33976451/>

ALABDULWAHHAB, Khalid M.; KAZMI, Syed Yousaf; SAMI, Waqas; et al. Use of online resources by undergraduate medical students at College of Medicine, Majmaah University, Kingdom of Saudi Arabia. PLOS ONE, v. I6, n. 8, p. e0255635, 2021. Acesso em: 12 de novembro de 2021< https://pubmed.ncbi.nlm.nih.gov/34347850/> 
ALSUYIHILI, Ali; MSHERGHI, Ahmed; ELHADI, Ahmed; et al. Impact of the COVID-i9 pandemic on medical education: Medical students' knowledge, attitudes, and practices regarding electronic learning. PLOS ONE, v. 15, n. II, p. e0242905, 2020. Acesso em: 12 de novembro de 2021 < https://pubmed.ncbi.nlm.nih.gov/33237962/>

ASHRAFZADEH, Sepideh; IMADOJEMU, Sotonye E.; VLEUGELS, Ruth Ann; et al. Strategies for effective medical student education in dermatology during the COVID-I9 pandemic. Journal of the American Academy of Dermatology, v. 84, n. I, p. e33-e34, 202I. Acesso em: 12 de novembro de 2021 < https://pubmed.ncbi.nlm.nih.gov/32828857/>

BACHIR, Bachir; NAJI, Amal; TFAYLI, Arafat. The educational and psychological impact of the COVID-I9 pandemic on medical students: A descriptive survey at the American University of Beirut. Medicine, v. Ioo, n. 28, p. e26646, 2021. Disponível em: <https://pubmed.ncbi.nlm.nih.gov/34260565/>. Acesso em: I2 nov. 2021.

BĄCZEK, Michał; ZAGAŃCZYK-BĄCZEK, Michalina; SZPRINGER, Monika; et al. Students' perception of online learning during the COVID-I9 pandemic: A survey study of Polish medical students. Medicine, v. Ioo, n. 7, p. e2482I, 2021. Acesso em: I2 de novembro de 2021 < https://pubmed.ncbi.nlm.nih.gov/33607848/>

BEHRENDS, Marianne; HOFFMANN, Ina; MARSCHOLLEK, Michael; et al. Teamwork, communication and exchange despite Covid-ı - experiences from a digital elective in human medicine studies as part of the HiGHmed project. GMS Journal for Medical Education; 37(7):Doc86, 2020. Disponível em: 〈https://www.egms.de/en/journals/zma/2020-37/zmaool379.shtml〉. Acesso em: I2 nov. 2021.

BINKS, Andrew P.; LECLAIR, Renée J.; WILLEY, Joanne M.; et al. Changing Medical Education, Overnight: The Curricular Response to COVID-19 of Nine Medical Schools. 
Teaching and Learning in Medicine, v. 33, n. 3, p.334-342, 2021. Disponível em: <https://pubmed.ncbi.nlm.nih.gov/33706632/>. Acesso em: I2 nov. 2021.

BLYTHE, Jenny; PATEL, Nimesh S. A.; SPIRING, Will; et al. Undertaking a high stakes virtual OSCE ("VOSCE") during Covid-ı. BMC Medical Education, v. 2I, n. I, p. 22I, 202I. Acesso em: 12 de novembro de 202I 〈https://pubmed.ncbi.nlm.nih.gov/33879139/>

Challenges of Online Medical Education in Pakistan During COVID-ig Pandemic. Journal of the College of Physicians and Surgeons Pakistan, v. 30, n. I, p. 67-69, 2020. Acesso em: 12 de novembro de 2021 < https://pubmed.ncbi.nlm.nih.gov/32723456/>

CHANDRASINGHE，P. C.; SIRIWARDANA，R. C.; KUMARAGE, S. K.; et al. A novel structure for online surgical undergraduate teaching during the COVID-I9 pandemic. BMC Medical Education, v. 20, n. I, p. 324, 2020. Disponível em: <https://pubmed.ncbi.nlm.nih.gov/32962691/>. Acesso em: I2 nov. 2021.

CHENG, Xin; CHAN, Lap Ki; PAN, San-Qiang; et al. Gross Anatomy Education in China during the Covid-ı Pandemic: A National Survey. Anatomical Sciences Education, v. I4, n. I, p. 8-18, 2021. Acesso em I2 de novembro de 202I < https://pubmed.ncbi.nlm.nih.gov/33217164/>

CUSCHIERI, Sarah; CALLEJA AGIUS, Jean. Spotlight on the Shift to Remote Anatomical Teaching During Covid-19 Pandemic: Perspectives and Experiences from the University of Malta. Anatomical Sciences Education, v. 13, n. 6, p.671-679, 2020. Disponível em: 〈https://pubmed.ncbi.nlm.nih.gov/32956579/>. Acesso em: I2 nov. 202I

DARNTON, Richard; LOPEZ, Tony; ANIL, Megha; et al. Medical students consulting from home: A qualitative evaluation of a tool for maintaining student exposure to patients during lockdown. Medical Teacher, v. 43, n. 2, p. 160-167, 2021. Acesso em: 12 de novembro de 2021< https://pubmed.ncbi.nlm.nih.gov/33045174/> 
DEDEILIA, Aikaterini; SOTIROPOULOS, Marinos G.; HANRAHAN, John Gerrard; et al. Medical and Surgical Education Challenges and Innovations in the COVID-I9 Era: A Systematic Review. In Vivo, v. 34, n. 3 suppl, p. I603-16II, 2020. Acesso em: I2 de novembro de 2021 < https://pubmed.ncbi.nlm.nih.gov/32503818/>

DOST, Samiullah; HOSSAIN, Aleena; SHEHAB, Mai; et al. Perceptions of medical students towards online teaching during the COVID-I9 pandemic: a national crosssectional survey of 2721 UK medical students. BMJ Open, v. Io, n. II, p. e042378, 2020. Disponível em <https://pubmed.ncbi.nlm.nih.gov/33154063/> Acesso em 12 de novembro de 202I.

FISCHBECK, Sabine; HARDT, Jochen; MALKEWITZ, Camila; et al. Evaluation of a digitized physician-patient-communication course evaluated by preclinical medical students: a replacement for classroom education? GMS Journal for Medical Education; 37(7):Doc85, 2020. Disponível em: <https://www.egms.de/en/journals/zma/202037/zmaoor378.shtml>. Acesso em: 12 nov. 2021.

GRZYCH, Guillaume; SCHRAEN-MASCHKE, Susanna. Interactive pedagogical tools could be helpful for medical education continuity during COVID-19 outbreak. Annales de Biologie Clinique, v. 78, n. 4, p. 446-448, 2020. Acesso em: 12 de novembro de 2021 https://pubmed.ncbi.nlm.nih.gov/3262773I/

HARENDZA, Sigrid; GÄRTNER, Julia; ZELESNIACK, Elena; et al. Evaluation of a telemedicine-based training for final-year medical students including simulated patient consultations, documentation, and case presentation. GMS Journal for Medical Education; 37(7):Doc94, 2020. Disponível em: 〈https://www.egms.de/en/journals/zma/202037/zmaoor387.shtml $>$. Acesso em: I2 nov. 2021.

HERRMANN-WERNER, Anne; ERSCHENS, Rebecca; ZIPFEL, Stephan; et al. Medical education in times of COVID-I9: survey on teachers' perspectives from a German medical faculty. GMS Journal for Medical Education; 38(5):Doc93, 2021. Disponível em: 
〈https://www.egms.de/en/journals/zma/202I-38/zmaoor489.shtml〉.

HU, Hai; XIAO, Yuanyuan; LI, Hao. The Effectiveness of a Serious Game Versus Online Lectures for Improving Medical Students' Coronavirus Disease 2019 Knowledge. Games for Health Journal, v. Io, n. 2, p. 139-I44, 2021. Acesso em: I2 de novembro de 2021< https://pubmed.ncbi.nlm.nih.gov/33434099/>

JUMREORNVONG, Oranicha; YANG, Emmy; RACE, Jasmine; et al. Telemedicine and Medical Education in the Age of COVID-19. Academic Medicine, v. 95, n. I2, p. 1838-1843, 2020. Acesso em: I2 de novembro de 2021 〈https://pubmed.ncbi.nlm.nih.gov/32889946/>

KANG, Ye Ji; KIM, Do-Hwan. Pre-clerkship students' perception and learning behavior of online classes during coronavirus disease 2019 pandemic. Korean Journal of Medical Education, v. 33, n. 2, p. I25-131, 2021. Acesso em:I2 de novembro de 2021 〈https://pubmed.ncbi.nlm.nih.gov/34062644/〉

KHALIL, Rehana; MANSOUR, Ali E.; FADDA, Walaa A.; et al. The sudden transition to synchronized online learning during the COVID-I9 pandemic in Saudi Arabia: a qualitative study exploring medical students' perspectives. BMC Medical Education, v. 20, n. I, p. 285, 2020. Acesso em: I2 de novembro de 202I < https://pubmed.ncbi.nlm.nih.gov/32859188/>

KIM, Ju Whi; MYUNG, Sun Jung; YOON, Hyun Bae; et al. How medical education survives and evolves during COVID-I9: Our experience and future direction. PLOS ONE, v. I5, n. I2, p. e0243958, 2020. Acesso em: I2 de novembro de 202I < https://pubmed.ncbi.nlm.nih.gov/33338045/>

KOVÁCS, Enikő; KÁLLAI, András; FRITÚZ, Gábor; et al. The efficacy of virtual distance training of intensive therapy and anaesthesiology among fifth-year medical students during the COVID-19 pandemic: a cross-sectional study. BMC Medical 
Education,

V. 2I,

n. I,

p. 393,

2021.

Disponível

em: <https://pubmed.ncbi.nlm.nih.gov/34294079/>. Acesso em: I2 nov. 2021.

LEE, I.R.; KIM, H.W.; LEE, Y.; et al. Changes in undergraduate medical education due to COVID-19: a systematic review. European Review for Medical and Pharmacological Sciences, $\quad$ v. $25, \quad$ n. 12, p. 4426-4434, 2021. Disponível em: 〈https://pubmed.ncbi.nlm.nih.gov/34227080/>. Acesso em: 12 nov. 2021.

MAGAlHÃES, Amanda Júlia de Arruda; ROCHA, Matheus Henrique Almeida; SANTOS, Samilla Cristinny; et al. O Ensino da Anamnese Assistido por Tecnologias Digitais durante a Pandemia da Covid-ı9 no Brasil. Revista Brasileira de Educação Médica, v. 44, n. suppl I, p. er63, 2020. Disponível em: 〈https://www.scielo.br/j/rbem/a/9VCdCPpP $3 \mathrm{NR}_{4} \mathrm{~S}_{2 n} \mathrm{Ykrh}$ 9qCD/?lang=pt . Acesso em: 12 nov. 202I.

MARSHALL, Ariela L.; WOLANSKYJ-SPINNER, Alexandra. COVID-ı: Challenges and Opportunities for Educators and Generation Z Learners. Mayo Clinic Proceedings, v. 95, n. 6, p. II35-II37, 2020. Acesso em: I2 de novembro de 2021 https://pubmed.ncbi.nlm.nih.gov/32376100/

MENDES, Karina Dal Sasso; SILVEIRA, Renata Cristina de Campos Pereira; GALVÃO, Cristina Maria. Use of the bibliographic reference manager in the selection of primary studies in integrative reviews. Texto \& Contexto - Enfermagem, v. 28, p. e20170204, 2019. Disponível em: 〈https://www.scielo.br/j/tce/a/HZD 4 WwnbqL8t7YZpdWSjypj/?lang=pt\#〉 Acesso em 25 novembro de 2021.

MORETTI-PIRES, Rodrigo Otávio et al. Estratégias pedagógicas na educação médica aos desafios de Covid-ı9: revisão de escopo. Rev. bras. educ. med. , Brasília, v. 45, n. I, eo25, 2021. Disponível em 〈http://www.scielo.br/scielo.php?script=sci_arttext\&pid=Soroo$55022021000100302 \& \operatorname{lng}=e n \& n r m=$ iso $>$. Acesso em 20 de março de 2021. 
NEWMAN, Noah A.; LATTOUF, Omar M. Coalition for medical education-A call to action: A proposition to adapt clinical medical education to meet the needs of students and other healthcare learners during COVID-19. Journal of Cardiac Surgery, v. 35, n. 6, p. II74-II75, 2020. Acesso em: I2 de novembro de 202I < https://pubmed.ncbi.nlm.nih.gov/32353907/>

OLADIPO, Aishat Temitope; FASHOLA, Oluwayemisi Tolulope; AGBOOLA, Eniola Ifedolapo; et al. Challenges with medical education in Nigeria in the COVID-I9 era. Pan African Medical Journal, v. 37, 2020. Disponível em: 〈https://www.panafrican-medjournal.com/content/article/37/223/full>. Acesso em: I2 nov. 2021.

OLMES, Gregor Leonhard; ZIMMERMANN, Julia Sarah Maria; STOTZ, Lisa; et al. Students' attitudes toward digital learning during the COVID-I9 pandemic: a survey conducted following an online course in gynecology and obstetrics. Archives of Gynecology and Obstetrics, v. 304, n. 4, p. 957-963, 2021. Acesso em I2 de novembro de 2021 < https://pubmed.ncbi.nlm.nih.gov/34355284/>

OSBORNE, Fiona; HARRISON, Miles; FISHER, James; et al. Using medical reality television as a technology-enhanced learning strategy to provide authentic patient care experiences during clinical placements: a case study research investigation. BMC Medical Education, v. 2I, n. I, p. I5, 202I. Acesso em: 12 de novembro de 202I < https://pubmed.ncbi.nlm.nih.gov/33407378/>

OSSAI, Edmund Ndudi. Impact of COVID-19 on medical education and the challenges: how prepared is Nigeria? The Pan African Medical Journal, v. 37, n. Suppl I, p. 45, 2020. Disponível em: 〈https://pubmed.ncbi.nlm.nih.gov/33552373/>. Acesso em: I2 nov. 202I.

OTAKI, Farah; ZAHER, Shroque; DU PLESSIS, Stefan; et al. Introducing the ${ }_{4} \mathrm{Ps}$ Model of Transitioning to Distance Learning: A convergent mixed methods study conducted during the COVID-19 pandemic. PLOS ONE, v. I6, n. 7, p. e0253662, 2021. Disponível em: < https://pubmed.ncbi.nlm.nih.gov/34264968/>. Acesso em: I2 nov. 2021. 
PARK, Hyunmi; LEE, Young-Mee; HO, Ming-Jung; et al. How the coronavirus disease 2019 pandemic changed medical education and deans' perspectives in Korean medical schools. Korean Journal of Medical Education, v. 33, n. 2, p. 65-74, 2021. Acesso em: 12 de novembro de 202I < https://pubmed.ncbi.nlm.nih.gov/33957729/>

PETTITT-SCHIEBER, Brian; KUO, Marissa; STEEHLER, Andrew; et al. Implementation and evaluation of eight virtual surgical electives for medical students during the COVID-I9 pandemic. The American Journal of Surgery, v. 222, n. 2, p. 248-253, 2021. Acesso em I2 de novembro de 2021< https://pubmed.ncbi.nlm.nih.gov/33558060/>

POLUJANSKI, Sabine; SCHINDLER, Ann-Kathrin; ROTTHOFF, Thomas. Academicassociated emotions before and during the COVID-I9-related online semester - a longitudinal investigation of first-year medical students. GMS Journal for Medical Education; 37(7):Doc77, 2020. $\quad$ Disponível em: 〈https://www.egms.de/static/en/journals/zma/2020-37/zmaoor370.shtml〉. Acesso em: 12 nov. 2021.

RAHM, Ann-Kathrin; TÖLLNER, Maximilian; HUBERT, Max Ole; et al. Effects of realistic e-learning cases on students' learning motivation during COVID-I9. PLOS ONE, v. I6, n. 4, p. e0249425, 2021. Acesso em I2 de novembro de 202I < https://pubmed.ncbi.nlm.nih.gov/33882079/>

REDINGER, Kathryn; GREENE, Jeffrey. Virtual Emergency Medicine Clerkship Curriculum during the COVID-19 Pandemic: Development, Application, and Outcomes. Western Journal of Emergency Medicine, v. 22, n. 3, 2021. Disponível em: 〈https://escholarship.org/uc/item/5xh8z53q〉. Acesso em: I2 nov. 2021.

REINHART, Anika; MALZKORN, Bastian; DÖING, Carsten; et al. Undergraduate medical education amid COVID-I9: a qualitative analysis of enablers and barriers to acquiring competencies in distant learning using focus groups. Medical Education Online, 
v. 26, n. I, p. 1940765, 2021. Disponível em: 〈https://pubmed.ncbi.nlm.nih.gov/34128776/>. Acesso em: I2 nov. 202I.

ROSENTHAL, Hailey; SIKKA, Neha; LIEBER, Adam; et al. A Near-Peer Educational Model for Online, Interactive Learning in Emergency Medicine. Western Journal of Emergency Medicine, v. 22, n. I, 2021. Disponível em: $\langle$ https://escholarship.org/uc/item/ojp6n8fs〉. Acesso em: I2 nov. 202I.

ROSKVIST, Rachel; EGGLETON, Kyle; GOODYEAR-SMITH, Felicity. Provision of e-learning programmes to replace undergraduate medical students' clinical general practice attachments during COVID-I9 stand-down. Education for Primary Care, v. 3I, n. 4, p. 247-254, 2020. Disponível em: 〈https://pubmed.ncbi.nlm.nih.gov/32469632/〉. Acesso em: 12 nov. 202I.

SANDHAUS, Yoram; KUSHNIR, Talma; ASHKENAZI, Shai. Electronic Distance Learning of Pre-clinical Studies During the COVID-19 Pandemic: A Preliminary Study of Medical Student Responses and Potential Future Impact. The Israel Medical Association journal: IMAJ, $\quad$ v. 22, $\quad$ n. 8, p. 489-493, 2020. Disponível em: 〈https://pubmed.ncbi.nlm.nih.gov/3323658I/>. Acesso em: I2 nov. 202I.

SANDHU, Preeti; DE WOLF, Maisie. The impact of COVID-I9 on the undergraduate medical curriculum. Medical Education Online, v. 25, n. I, p. 1764740, 2020. Acesso em: I2 de novembro de 2021 < https://pubmed.ncbi.nlm.nih.gov/33154063/>

SANTOS, Bruna Mascarenhas et al . Educação Médica durante a Pandemia da Covid-ı: uma Revisão de Escopo. Rev. bras. educ. med., Brasília , v. 44, supl. I, e139, 2020. Disponível em: $\quad$ http://www.scielo.br/scielo.php?script=sci_arttext\&pid=Soroo$55022020000500301 \& \operatorname{lng}=$ en $\&$ nrm=iso>. Acesso em: i9 Mar. 2021.

SARKAR, S; MISHRA, P; NAYAK, A. Online open-book examination of undergraduate medical students - a pilot study of a novel assessment method used during the coronavirus 


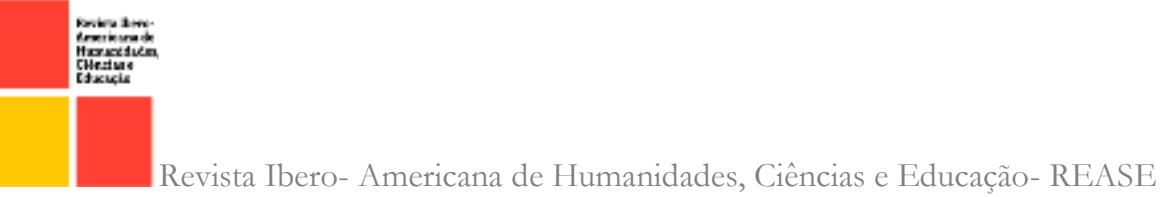

disease 2019 pandemic. The Journal of Laryngology \& Otology, v. 135, n. 4, p. 288-292, 2021. Acesso em: I2 de novembro de 2021 <https://pubmed.ncbi.nlm.nih.gov/33413724/>

SHAHRVINI, Bita; BAXTER, Sally L.; COFFEY, Charles S.; et al. Pre-clinical remote undergraduate medical education during the COVID-I9 pandemic: a survey study. BMC Medical Education, v. 2I, n. I, p. I3, 202I. Disponível em: <https://pubmed.ncbi.nlm.nih.gov/33407376/>. Acesso em: I2 nov. 2021.

SHARMA, Divyansh; BHASKAR, Sonu. Addressing the Covid-19 Burden on Medical Education and Training: The Role of Telemedicine and Tele-Education During and Beyond the Pandemic. Frontiers in Public Health, v. 8, p. 589669, 2020. Acesso em: I2 de novembro de 2021 < https://pubmed.ncbi.nlm.nih.gov/33330333/>

SITU-LACASSE, Elaine; ACUÑA, Josie; HUYNH, Dang; et al. Can ultrasound novices develop image acquisition skills after reviewing online ultrasound modules? BMC Medical Education, v. 2I, n. I, p. 175, 202I. Acesso em: I2 de novembro de 202I < https://pubmed.ncbi.nlm.nih.gov/3374368o/>

SONG, Yaxiang; WANG, Shu; LIU, Yixian; et al. Online education at the medical School of Tongji University during the COVID-I9 pandemic: a cross-sectional study. BMC Medical Education, v. 2I, n. I, p. 512, 2021. Acesso em 12 de novembro de 2021 〈https://pubmed.ncbi.nlm.nih.gov/3458370o/>

SUKUMAR, Smrithi; ZAKARIA, Adam; LAI, Cindy J.; et al. Designing and Implementing a Novel Virtual Rounds Curriculum for Medical Students' Internal Medicine Clerkship During the COVID-i9 Pandemic. MedEdPORTAL, p. IIIo6, 202I. Acesso em I2 de novembro de 2021 < https://pubmed.ncbi.nlm.nih.gov/33768143/> THOM, Mitchell L.; KIMBLE, Blair A.; QUA, Kelli; et al. Is remote near-peer anatomy teaching an effective teaching strategy? Lessons learned from the transition to online learning during the Covid-19 pandemic. Anatomical Sciences Education, v. I4, n. 5, p. 552- 
56I, 2021. Disponível em:〈https://pubmed.ncbi.nlm.nih.gov/34268899/〉. Acesso em: I2 nov. 202I.

TRIEMSTRA, Justin D.; HAAS, Mary R.C.; BHAVSAR-BURKE, Indira; et al. Impact of the COVID-ı9 Pandemic on the Clinical Learning Environment: Addressing Identified Gaps and Seizing Opportunities. Academic Medicine, v. 96, n. 9, p. 1276-1281, 2021. Acesso em: I2 de novembro de 2021< https://pubmed.ncbi.nlm.nih.gov/34432665/>

ZOTTMANN, Jan M.; HORRER, Anna; CHOUCHANE, Amir; et al. Isn't here just there without a " $\mathrm{t}$ " - to what extent can digital Clinical Case Discussions compensate for the absence of face-to-face teaching? GMS Journal for Medical Education; 37(7):Doc99, 2020. Disponível em: 〈https://www.egms.de/en/journals/zma/2020-37/zmaoor392.shtml〉. Acesso em: 12 novembro de 2021. 